\title{
REVIEW
}

\section{Strongyloidiasis - the most neglected of the neglected tropical diseases?}

\author{
Annette Olsen ${ }^{a, *}$, Lisette van Lieshout ${ }^{b}$, Hanspeter Marti $^{c}$, Ton Polderman ${ }^{b}$, \\ Katja Polman ${ }^{d}$, Peter Steinmann ${ }^{\text {e,f }}$, Russell Stothard", Søren Thybo ${ }^{h}$, \\ Jaco J. Verweij ${ }^{b}$, Pascal Magnussen ${ }^{a}$
}

\footnotetext{
a DBL-Centre for Health Research and Development, University of Copenhagen, Copenhagen, Denmark

${ }^{\mathrm{b}}$ Department of Parasitology, Leiden University Medical Center, Leiden, The Netherlands

c Department of Medical and Diagnostic Services, Swiss Tropical Institute, Basel, Switzerland

d Department of Parasitology, Prince Leopold Institute of Tropical Medicine, Antwerp, Belgium

e Department of Public Health and Epidemiology, Swiss Tropical Institute, Basel, Switzerland

${ }^{f}$ National Institute of Parasitic Diseases, China CDC, Shanghai, People's Republic of China

g Department of Zoology, Natural History Museum, London, UK

h Department of Epidemiology, Rigshospitalet, Copenhagen, Denmark
}

Received 29 December 2008; received in revised form 17 February 2009; accepted 17 February 2009 Available online 27 March 2009

\section{KEYWORDS \\ Strongyloides \\ stercoralis; \\ Diagnosis; \\ Morbidity; \\ Treatment; \\ Control; \\ Neglected tropical \\ disease}

\begin{abstract}
Summary Soil-transmitted helminths of the genus Strongyloides (S. fuelleborni and the more prevalent S. stercoralis) are currently believed to infect an estimated 30-100 million people worldwide. The health consequences of $S$. stercoralis infections range from asymptomatic light infections to chronic symptomatic strongyloidiasis. Uncontrolled multiplication of the parasite (hyperinfection) and potentially life-threatening dissemination of larvae to all internal organs is found among individuals with compromised immune system functions. This paper provides an overview of the current state of the art in relation to diagnostic methods for detecting the infection, the morbidity caused by the infection and the recommended treatment. It further discusses some of the reasons why this infection is so neglected and the consequence of this for the estimated global prevalence. The paper finally points to the gaps in our knowledge and future research needs related to this infection. As Strongyloides infections have the potential to develop into severe disease in certain population subgroups, untreated infections could cause serious problems in the community. Therefore, we need to carefully investigate this parasite in order to develop and implement effective control programmes.

(c) 2009 Royal Society of Tropical Medicine and Hygiene. Published by Elsevier Ltd. All rights reserved.
\end{abstract}

\footnotetext{
* Corresponding author.

E-mail address: aol@life.ku.dk (A. Olsen).
} 


\section{Introduction}

Soil-transmitted helminths of the genus Strongyloides currently infect an estimated 30-100 million people worldwide. ${ }^{1}$ At least two species parasitize humans S. fuelleborni and the more prevalent S. stercoralis. ${ }^{2,3}$ Strongyloides fuelleborni is a zoonosis that usually occurs in non-human primates, but human infections occur in Africa and Southeast Asia. Strongyloides stercoralis is an intestinal nematode that infects humans percutaneously. It is common in many areas where sanitary conditions are poor, ${ }^{4}$ and where the climate is warm and humid. However, the parasite is also endemic in several subtropical and temperate regions, e.g. southern areas of the USA, Europe and Japan. The ability of $S$. stercoralis to replicate within the host (autoinfection) leads to persistent infections and can result in chronic disease with a wide variation of clinical manifestations. Uncontrolled multiplication of the parasite (hyperinfection) and potentially life-threatening dissemination of larvae to all internal organs are found among individuals with compromised immune system functions. Apart from parasitizing the vertebrate intestine, the life cycle of these nematodes can also include one or more (depending on the species) free-living generations, leading to important epidemiological consequences.

At the Third Global Meeting of the Partners for Parasite Control, held at WHO headquarters in November 2004, it was recommended that measures for the control of Strongyloides should be included in health packages targeting areas where the infection is endemic. ${ }^{5}$ Today, more than 4 years later, no action has been taken and no specific measures have been implemented. One of the probable reasons is the lack of even basic data on Strongyloides endemicity and epidemiology. We also do not know to what extent existing soil-transmitted helminth and lymphatic filariasis control programmes affect the epidemiology of Strongyloides, as the demonstration of this parasite requires special detection methods, which usually fall outside the scope of these programmes.

As Strongyloides infections have the potential to develop into severe disease in certain population subgroups, untreated infections could cause serious problems in the community. We therefore feel that we have to take action now and carefully investigate this parasite in order to develop and implement effective control programmes.

\section{Presentation of the parasite}

The genus Strongyloides contains more than 50 species with an almost equally wide range of hosts. At least two species are known to infect humans.

Strongyloides fuelleborni primarily infects various primates in Africa and Southeast Asia but has also been found in humans. Thus, the parasite is zoonotic or, at least, zoonotic in its origin. ${ }^{6}$ Strongyloides fuelleborni eggs can be found in stool and although the question of autoinfection is still not resolved, it probably does not occur in persons with egg-passing infections. ${ }^{6}$

The cosmopolitan S. stercoralis occurs in over 70 subtropical and tropical countries, but is also endemic in several temperate countries where conditions are favourable. For example, S. stercoralis infections are, together with hookworm infections, the only officially recognized occupational parasitic health hazard for miners in Germany. ${ }^{7}$ The parasite infects humans, other primates and dogs. Strongyloides stercoralis has a complex life cycle including a direct, an autoinfective and a non-parasitic free-living developmental cycle (Figure 1). Only parasitic females are found in the host where they are embedded in the submucosa of the duodenum and parthenogenetically produce dozens of embryonated eggs a day. These hatch in the gut lumen of the host and the first-stage larvae are passed out in faeces and either develop into infective third-stage larvae or into freeliving adult males and females. Alternatively, larvae may develop to the third stage still within the gastrointestinal tract and penetrate the intestinal mucosa or perianal skin, restarting a new infection cycle without ever leaving their host. The free-living adults reproduce sexually and the offspring develop into infective third-stage larvae. There exists only one free-living generation of S. stercoralis. ${ }^{8}$ Third-stage larvae are able to penetrate the skin of the human host, reach the lungs via the blood circulation and enter the respiratory pathways, from where they migrate upwards through the trachea to be swallowed and finally reach the small intestine where they mature into adult egg-laying females.

\section{Diagnosis and global epidemiology of Strongyloides}

Standard parasitological diagnostic methods for detecting $S$. stercoralis infection are concentration techniques or stool culture methods. ${ }^{9}$ However, these methods are laborious, time consuming, and, in the case of stool cultures, need well trained technicians in order to differentiate $S$. stercoralis from hookworm larvae. Several immunological tests, such as ELISA, IFAT and Western blot, have also been described for the diagnosis of strongyloidiasis. ${ }^{10,11}$ These assays show variable sensitivity and specificity depending on antigen preparation and immunoglobulin isotypes used, and on the population tested. In specific target populations, such as immigrants to non-endemic regions, serology has proven to be a valuable tool for screening and for the evaluation of therapies; ${ }^{12}$ however, serology seems to lack sufficient sensitivity to diagnose recently acquired infections. ${ }^{13}$ Although serology seems to have good negative predictive value if used in endemic areas, ${ }^{14}$ specificity appears to be more problematic as pronounced crossreactivity is observed with other helminths, especially filaria. Specific detection of Strongyloides DNA in human stool samples by real-time PCR has recently been suggested to be a promising alternative diagnostic approach, but needs further exploration.

The current estimate of 30-100 million Strongyloides infections in humans ${ }^{1}$ is likely to be too low. To achieve a reliable estimate of the true prevalence of infection, several stool samples should be collected over consecutive days and tested by various diagnostic methods. In a populationbased study in China, combining diagnostic approaches and repeated examinations resulted in an unexpectedly high prevalence of $11.7 \% .^{15}$ Using a similar approach, Strongyloides was shown to be a very common parasite (prevalence $10.6 \%$ ) in northern Ghana. ${ }^{16}$ In a recent study in Mozambique (unpublished data) careful examinations of direct smears 


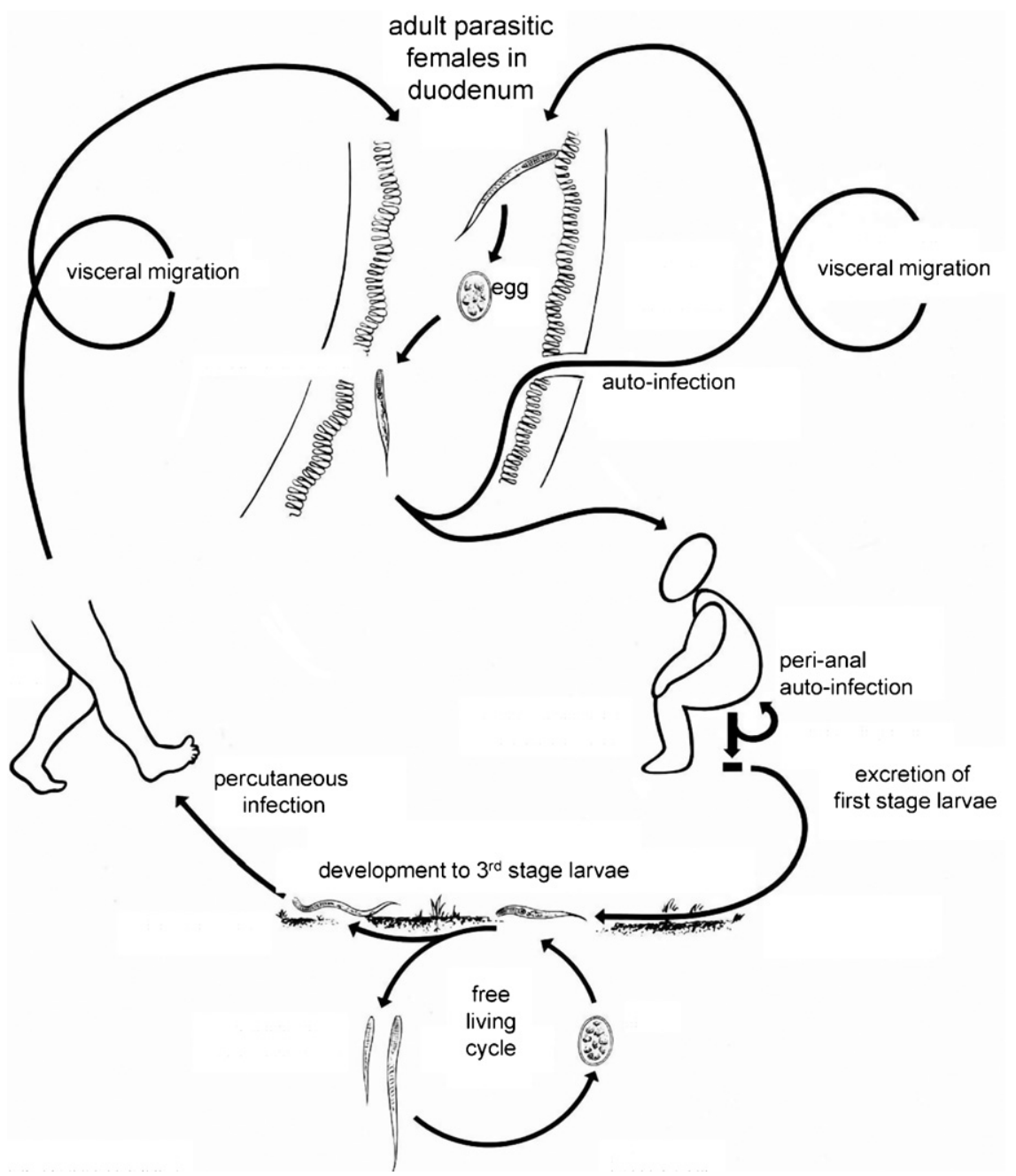

Figure 1 The life cycle of Strongyloides stercoralis. Drawing by A.M. Polderman.

and formol ether concentration sediments showed $9 \%$ and $12 \%$ of the population to be infected, while a combination of culture, Baermann concentration and PCR revealed a prevalence of $48 \%$.

\section{Morbidity caused by Strongyloides}

The health consequences of $S$. stercoralis infections range from asymptomatic light infections to chronic symptomatic strongyloidiasis involving anything from mild gastrointestinal morbidity to severe life-threatening conditions. The pathology due to chronic uncomplicated S. stercoralis infections includes pruritus, skin eruptions, larva currens, abdominal pain, diarrhoea, nausea and weight loss. ${ }^{17}$

The most severe effects of Strongyloides infections occur in patients receiving immunosuppressive therapy with corticosteroids. ${ }^{18-21}$ In these patients, the immune system is no longer able to keep the larvae emerging from the autoinfective cycle under control, resulting in hyperinfection affecting the proper functioning of the gastrointestinal tract or the dissemination of larvae to multiple organs including the brain. Such massive infections may lead to life-threatening conditions with pneumonia, meningitis, septicaemia and intestinal obstruction. ${ }^{17}$ The mortality rate of disseminated infections has been estimated to be as high as $87 \% .^{22}$ Immunosuppression with Cyclosporine A is not known to have such devastating effects. ${ }^{23}$

In many parts of the world strongyloidiasis occurs together with HIV/AIDS, but co-infection has not been found to be associated with higher $S$. stercoralis prevalence or the development of hyperinfections. ${ }^{24}$ However, S. stercoralis hyperinfection has been described in AIDS patients as part of the immune reconstitution syndrome after starting highly active antiretroviral therapy. ${ }^{25}$ In a study in Tanzania involving HIV-infected pregnant women, S. stercoralis infection was associated with a higher risk of low birth weight, which is the single most important factor for high infant mortality. ${ }^{26}$ In contrast, human T cell lymphotropic virus type 1 infection is strongly associated with a higher $S$. stercoralis prevalence as well as with an increased incidence of hyperinfection. ${ }^{27-29}$

Although there is a pronounced association between disseminated strongyloidiasis and suppression of the immune system, there are also a significant number of fatal cases in the literature which could not be linked to any known impairment of the immune system. ${ }^{30}$ They have tentatively been explained by host factors of intestinal parasite 
control which act at the interface between the host and the parasite, i.e. the mucosa of the gastrointestinal tract, and are mediated by some local control mechanisms. In impoverished populations where subclinical malnutrition is common, profound morphological disruption of the small intestinal mucosa is highly prevalent. These changes may have functional repercussions on the hosts' ability to fight off infections. ${ }^{31}$ This hypothesis might also explain why severe complicated strongyloidiasis with no clinical evidence of immunosuppression is more frequently observed in persons living in developing countries. Malnutrition, one of the most important causes of immunodeficiency in developing countries, was found to be associated with severe strongyloidiasis. ${ }^{30}$ Autoimmune diseases such as diabetes mellitus may exacerbate complications and impact on the severity of Strongyloides infection. ${ }^{32}$

\section{Treatment}

The recommended treatment for strongyloidiasis is either ivermectin $(200 \mu \mathrm{g} / \mathrm{kg}$ body weight in a single dose) or albendazole (400 mg daily for 3 days). ${ }^{33,34}$ In a study in Zanzibar, the effect of the two regimens was compared. A cure rate of $82.9 \%$ was achieved for ivermectin while three doses of albendazole cured $45.0 \%$ of the infected individuals. ${ }^{33}$

Most helminth control programmes employ mass drug administration as their principal tool; hence, they offer treatment without prior diagnosis. For control of soiltransmitted helminths, the target population is usually schoolchildren, but regular (e.g. annual) mass treatment of entire communities using one of the four common antehelminthic compounds included in the WHO model list of essential drugs is recommended wherever the prevalence is $>50 \% .{ }^{35}$ In areas where schistosomiasis is co-endemic with soil-transmitted helminths, praziquantel $(40 \mathrm{mg} / \mathrm{kg}$ body weight) is co-administered with albendazole $(400 \mathrm{mg}$ single dose) or mebendazole (500 mg single dose), again mainly to schoolchildren. In areas targeted for lymphatic filariasis control, albendazole ( $400 \mathrm{mg}$ single dose) and ivermectin $(150 \mu \mathrm{g} / \mathrm{kg}$ body weight) or diethylcarbamazine (where onchocerciasis is not endemic) are given to the entire population above 4 years old. To minimize costs, collaboration between the programmes is encouraged and is currently implemented in many countries. ${ }^{36}$ Thus, in areas where schistosomiasis and lymphatic filariasis are both endemic, albendazole plus ivermectin followed by praziquantel 1 week later is the preferred regimen. Among all these regimens, only the distribution of ivermectin in the frame of lymphatic filariasis control can be expected to result in a meaningful reduction of the prevalence of S. stercoralis. In areas with strongyloidiasis, targeted chemotherapy of high risk groups should be considered. As a minimum, all patients scheduled to receive corticosteroids should be given preventive anthelminthic treatment if they live in or have travelled in potential endemic areas. ${ }^{21}$

\section{Why is Strongyloides infection so neglected?}

The prevalence of S. stercoralis is thought to be severely underestimated due to the low sensitivity of the currently available diagnostic tools and a dearth of specialized surveys. The most widely used method for the identification of human intestinal helminth infections in developing countries, i.e. the Kato-Katz technique, detects eggs in faeces but fails to demonstrate larvae. The performance of standard procedures like formol-ether concentration methods is limited by the low concentration of larval stages in faecal specimens in chronic strongyloidiasis. Parasite-specific concentration methods like the Baermann technique and culture methods ${ }^{9}$ are much more sensitive, but even with the use of these methods many light (chronic) infections are missed. Therefore, S. stercoralis infections are seldom detected and many areas without information persist on the global S. stercoralis distribution map. Thus, the epidemiological knowledge regarding this parasite is patchy at best. Only in patients with disseminated strongyloidiasis may large numbers of larvae be demonstrated in faeces, sputum, urine and lung fluids as well as in organ biopsies. Chronic uncomplicated S. stercoralis infections also cause subtle pathology, which is non-specific for this infection. Therefore, the infection often remains undiagnosed.

\section{Gaps in knowledge - future research needs}

It is encouraging that the Partners for Parasite Control recommend that Strongyloides infections should be included in health packages wherever it is endemic. ${ }^{5}$ Today, the infection is only targeted passively - and, presumably, not effectively - in the framework of integrated helminth control programmes. The notion that an additional parasite can be controlled by these programmes may lead to a better cost-effectiveness rating of such programmes and, once the epidemiology and public health significance of strongyloidiasis has been determined, to better acceptance. Thus, the specific impact of these programmes on S. stercoralis awaits careful evaluation. For example, we do not know how the currently used drug regimes influence the course of infection. The drug dosages are suboptimal especially in areas where lymphatic filariasis is not endemic and therefore ivermectin is not used. One dose of albendazole will usually not clear the infection. This is of particular concern because the parasite replicates within the host. The residual parasites may represent a proportion of the population which is more refractory to the drug. It is therefore possible that resistance will develop and become established.

Many epidemiological aspects of Strongyloides infection are unknown or only poorly understood. We do not know in detail where Strongyloides is endemic, which prevalence rates and intensities can typically be expected in different settings and populations, the zoonotic aspects, geographical variations, e.g. in transmission and clinical presentation, host immunity and the risk factors for dissemination, and social, cultural, ecological and environmental aspects.

Improvement and simplification is needed in the conventional diagnostic methods, to facilitate epidemiological studies of S. stercoralis in the field. There also is an urgent need to employ modern molecular methods to improve diagnosis and for the differentiation between species and strains from different hosts and geographical origin. It should also be established if we are dealing with a species complex or clearly defined biological species. 
Little is known about the true clinical relevance of Strongyloides infections, the symptoms associated with different infection patterns, the association between Strongyloides and other infectious diseases, and of the relationship between severe infections and autoimmune diseases such as diabetes. The relevance of malnutrition for Strongyloides, especially among children, and the effects of the resulting state on the clinical outcome of S. stercoralis infection as well as implications for pregnant women still need to be revealed.

It is uncommon for helminths to kill their host and in healthy individuals, $\mathrm{S}$. stercoralis usually causes little or 'only' subtle pathology, allowing the infection to remain undiagnosed - and untreated - for years. Thus, the parasite can persist in the host, perpetuating parasite dispersal and the risk of infection among the community. Given the tendency to develop severe disease in certain population subgroups, S. stercoralis is of major public health concern. Furthermore, since the use of immunosuppressive therapy becomes increasingly common in transition economies like China, India, Southeast Asia and Latin America, Strongyloides could cause serious problems in the future if we do not take action now. We need to carefully investigate this parasite, develop control programmes and implement them where appropriate.

\section{Funding: None.}

Conflicts of interest: None declared.

Ethical approval: Not required.

\section{References}

1. Bethony J, Brooker S, Albonico M, Geiger SM, Loukas A, Diemert $D$, et al. Soil-transmitted helminth infections: ascariasis, trichuriasis, and hookworm. Lancet 2006;367:1521-32.

2. Speare P. Identification of species of Strongyloides. In: Grove $\mathrm{DI}$, editor. Strongyloidiasis - a major roundworm infection of man. London: Taylor \& Francis; 1989. p. 11-83.

3. Viney ME, Lok JB. Strongyloides spp. WormBook 2007 May: $1-15$.

4. Hall A, Conway DJ, Anwar KS, Rahman ML. Strongyloides stercoralis in an urban slum community in Bangladesh: factors independently associated with infection. Trans $R$ Soc Trop Med Hyg 1994;88:527-30.

5. WHO. Deworming for Health and Development. Report of the third global meeting of the partners for parasite control. Geneva: World Health Organization; 2005. WHO/CDS/PVC/2005.14.

6. Ashford RW, Barnish G. Strongyloides fuelleborni and similar parasites in animals and man. In: Grove DI, editor. Strongyloidiasis - a major roundworm infection of man. London: Taylor \& Francis; 1989. p. 271-86.

7. Bundesanstalt für Arbeitsschutz und Arbeitsmedizin (BauA). Berufskrankheiten-Verordnung (BKV) vom 31. Oktober 1997 (BGBI. I S. 2623), zuletzt geändert durch die Verordnung vom 5. September 2002 (BGBl. I S. 3541). Dortmund: BauA; 23 March 2004. http://www.baua.de/de/Themen-vonA-Z/Berufskrankheiten/Rechtsgrundlagen/BKV.html [accessed 17 February 2009].

8. Streit A. Reproduction in Strongyloides (Nematoda): a life between sex and parthenogenesis. Parasitology 2008;135: 285-94.
9. Cheesbrough M. Medical laboratory manual for tropical countries, Vol. 1, 2nd ed Cambridge: Tropical Health Technology and Butterworths; 1987.

10. Silva LP, Barcelos ISC, Passos-Lima AB, Espindola FS, Campos DMB, Costa-Cruz JM. Western blotting using Strongyloides ratti antigen for the detection of IgG antibodies as confirmatory test in human strongyloidiasis. Mem Inst Oswaldo Cruz 2003;98:687-91.

11. Rodrigues RM, De Oliveira MC, Sopelete MC, Silva DA, Campos DM, Taketomi EA, et al. IgG1, IgG4, and IgE antibody responses in human strongyloidiasis by ELISA using Strongyloides ratti saline extract as heterologous antigen. Parasitol Res 2007;101:1209-14.

12. Page WA, Dempsey K, McCarthy JS. Utility of serological followup of chronic Strongyloides after anthelminthic chemotherapy. Trans $R$ Soc Trop Med Hyg 2006;100:1056-62.

13. Sudarshi S, Stumpfle R, Armstrong M, Ellman T, Parton S, Krishnan $P$, et al. Clinical presentation and diagnostic sensitivity of laboratory tests for Strongyloides stercoralis in travellers compared with immigrants in a non-endemic country. Trop Med Int Health 2003;8:728-32.

14. Yori PP, Kosek M, Gilman RH, Cordova J, Bern C, Chavez CB, et al. Seroepidemiology of strongyloidiasis in the Peruvian Amazon. Am J Trop Med Hyg 2006;74:97-102.

15. Steinmann P, Zhou XN, Du ZW, Jiang JY, Wang LB, Wang XZ, et al. Occurrence of Strongyloides stercoralis in Yunnan Province, China, and comparison of diagnostic methods. PLoS Negl Trop Dis 2007; 1:e75.

16. Yelifari L, Bloch P, Magnussen P, van Lieshout L, Dery G, Anemana $S$, et al. Distribution of human Oesophagostomum bifurcum, hookworm and Strongyloides stercoralis infections in northern Ghana. Trans $R$ Soc Trop Med Hyg 2005;99:32-8.

17. Grove DI. Clinical manifestations. In: Grove DI, editor. Strongyloidiasis - a major roundworm infection of man. London: Taylor \& Francis; 1989. p. 155-73.

18. Morgan JS, Schaffner W, Stone WJ. Opportunistic strongyloidiasis in renal transplant recipients. Transplantation 1986;42:518-24.

19. Ghosh K, Ghosh K. Strongyloides stercoralis septicaemia following steroid therapy for eosinophilia: report of three cases. Trans $R$ Soc Trop Med Hyg 2007; 101:1163-5.

20. Marcos LA, Terashima A, DuPont HL, Gotuzzo E. Strongyloides hyperinfection syndrome: an emerging global infectious disease. Trans $R$ Soc Trop Med Hyg 2008;102:314-8.

21. Fardet L, Généreau T, Cabane J, Kettaneh A. Severe strongyloidiasis in corticosteroid-treated patients. Clin Microbiol Infect 2006;12:945-7.

22. Siddiqui AA, Berk SL. Diagnosis of Strongyloides stercoralis infection. Clin Infect Dis 2001;33:1040-7.

23. Grove DI. Treatment. In: Grove DI, editor. Strongyloidiasis - a major roundworm infection of man. London: Taylor \& Francis; 1989. p. 199-231.

24. Viney ME, Brown M, Omoding NE, Bailey JW, Gardner MP, Roberts $E$, et al. Why does HIV infection not lead to disseminated strongyloidiasis? J Infect Dis 2004;190:2175-80.

25. Brown M, Cartledge JD, Miller RF. Dissemination of Strongyloides stercoralis as an immune restoration phenomenon in an HIV-1-infected man on antiretroviral therapy. Int J STD AIDS 2006;17:560-1.

26. Dreyfuss ML, Msamanga GI, Spiegelman D, Hunter DJ, Urassa EJN, Hertzmark E, et al. Determinants of low birth weight among HIV-infected pregnant women in Tanzania. Am J Clin Nutr 2001;74:814-26.

27. Carvalho EM, Da Fonseca Porto A. Epidemiological and clinical interaction between HTLV-1 and Strongyloides stercoralis. Parasite Immunol 2004;26:487-97.

28. Keiser PB, Nutman TB. Strongyloides stercoralis in the immunocompromised population. Clin Microbiol Rev 2004;17:208-17. 
29. Hirata T, Uchima N, Kishimoto K, Zaha O, Kinjo N, Hokama A, et al. Impairment of host immune response against Strongyloides stercoralis by human cell lymphotropic virus type 1 infection. Am J Trop Med Hyg 2006;74:246-9.

30. Genta RM. Immunology. In: Grove DI, editor. Strongyloidiasis a major roundworm infection of man. London: Taylor \& Francis; 1989. p. 133-53.

31. Bundy DAP, Golden MHN. The impact of host nutrition on gastrointestinal helminth populations. Parasitology 1987; $95: 523-35$.

32. Mendonça SCL, Gonçalves-Pires MRF, Rodrigues RM, Ferreira Jr ÁF, Costa-Cruz JM. Is there an association between positive Strongyloides stercoralis serology and diabetes mellitus? Acta Trop 2006;99:102-5.

33. Marti H, Haji HJ, Savioli L, Chwaya HM, Mgeni AF, Ameir JS, et al. A comparative trial of a single-dose ivermectin versus three days of albendazole for treatment of Strongyloides stercoralis and other soil-transmitted helminth infections in children. Am J Trop Med Hyg 1996;55:477-81.

34. WHO. Prevention and control of schistosomiasis and soiltransmitted helminthiasis. Geneva: World Health Organization; 2002. Technical Report Series No. 912.

35. WHO. Preventive chemotherapy in human helminthiasis. Coordinated use of anthelminthic drugs in control interventions: a manual for health professionals and programme managers. Geneva: World Health Organization; 2006.

36. Olsen A. Efficacy and safety of drug combinations in the treatment of schistosomiasis, soil-transmitted helminthiasis, lymphatic filariasis and onchocerciasis. Trans $R$ Soc Trop Med Hyg 2007; 101:747-58. 